\title{
COMPOSICIÓN DE CARNE DE CERDO EN UN SISTEMA DE PRODUCCIÓN NATURAL
}

\section{COMPOSITION OF PORK MEAT IN A NATURAL PRODUCTION SYSTEM}

\author{
Valeria Velasco ${ }^{1 *}$, Victoria Vera ${ }^{1}$, Fernando Bórquez ${ }^{1}$, Pamela Williams ${ }^{1}$, Manuel Faúndez ${ }^{2}$, \\ Julio Alarcón-Enos ${ }^{3}$
}

\footnotetext{
${ }^{1}$ Departamento de Producción Animal, Facultad de Agronomía, Universidad de Concepción, Casilla 537, Chillán, Chile.

${ }^{2}$ Departamento de Producción Vegetal, Facultad de Agronomía, Universidad de Concepción, Casilla 537, Chillán, Chile.

${ }^{3}$ Laboratorio de Síntesis y Biotransformación de Productos Naturales, Departamento de Ciencias Básicas, Casilla 447, Universidad del Bío-Bío, Chillán, Chile

*Corresponding author E-mail: vvelasco@udec.cl
}

\section{RESUMEN}

La producción porcina en Chile se basa principalmente en un sistema de producción intensiva en confinamiento (sistema convencional). Sin embargo, en los últimos años, con el creciente interés por productos sanos, una alternativa atractiva es el sistema de producción natural, que es un sistema extensivo con acceso a pradera. El objetivo de este estudio fue determinar la composición de carne de cerdo producido en un sistema natural. Se determinó el $\mathrm{pH}$ y análisis proximal de: lomo (Longissimus dorsi) $(n=54)$ y pulpa de pierna $(n=54)$. Se determinó el perfil de ácidos grasos en lomo, pulpa de pierna y grasa de cobertura. El pH de la carne inicial estuvo entre 6,37 y 6,39 $(\mathrm{P}>0,05)$, disminuyendo a las 24 h post mortem a 5,81 y 5,70 , respectivamente $(P \leq 0,05)$. El contenido de materia seca de la carne fue cercano al $30 \%$, siendo levemente mayor en pulpa de pierna $(P \leq 0,05)$. No se presentaron diferencias significativas en los otros componentes analizados en la carne de cerdo $(P>0,05)$. La carne y la grasa de cobertura presentaron un mayor contenido de ácido oleico (18:1n9c), seguido de ácido palmítico $(16: 0)$ y esteárico $(18: 0)(P \leq 0,05)$. En la carne, el contenido de ácidos grasos saturados y monoinsaturados fue mayor, y el de poliinsaturados menor que en la grasa de cobertura $(P \leq 0,05)$. La carne de cerdo producido en sistema natural presenta características de calidad atractivas desde el punto de vista nutricional.

Palabras claves: pastoreo, calidad de carne, $\mathrm{pH}$, perfil de ácidos grasos, lomo de cerdo, pulpa de pierna de cerdo.

\section{ABSTRACT}

Swine production in Chile is mainly based on an intensive confinement production system (conventional system). However, in recent years, increasing interest for healthy food has resulted in the use of a natural production system, which is an attractive alternative consisting of an extensive system with access to grassland. The aim of this study was to determine the composition of pork meat produced in a natural system. The $\mathrm{pH}$ and proximal analysis of: pork loin (Longissimus dorsi) $(n=54)$ and pork leg pulp $(n=54)$ were determined. The profile of fatty acids in loin, leg pulp and

\footnotetext{
Recibido: 21 marzo 2019. $\quad$ Aceptado: 13 junio 2019.
} 
backfat was determined. The initial $\mathrm{pH}$ of pork meat was between 6.37 and $6.39(\mathrm{P}>0.05)$, decreasing at $24 \mathrm{~h}$ post mortem to 5.81 and 5.70 , respectively $(\mathrm{P} \leq 0.05)$. The dry matter content of pork meat was close to $30 \%$, being slightly higher in pork leg pulp $(\mathrm{P} \leq 0.05)$. There were no significant differences in the other components analyzed in pork meat $(P>0.05)$. The meat and backfat had a higher content of oleic acid (18:1n9c), followed by palmitic acid (16:0) and stearic acid (18:0) ( $\mathrm{P} \leq 0.05)$. In pork meat, the content of saturated and monounsaturated fatty acids was higher, and that of polyunsaturated fatty acids was lower than in the backfat $(P \leq 0.05)$. Therefore, pork meat from pigs raised in a natural production system has quality characteristics that are attractive from a nutritional point of view.

Key words: grazing, meat quality, $\mathrm{pH}$, fatty acid profile, pork loin, pork leg pulp.

\section{INTRODUCCION}

En Chile, la producción de carne el año 2018 alcanzó 1.501.055 toneladas, situándose en segundo lugar la carne de cerdo con 534.024 toneladas, después de la carne de ave, con un crecimiento del 7,6\% con respecto al año anterior. El consumo per cápita de carne de cerdo alcanzó $21 \mathrm{~kg}$ al año, ocupando el tercer lugar entre las carnes consumidas en el país (ASPROCER, 2019; ODEPA, 2019). La producción porcina en Chile se basa principalmente en un sistema de producción intensivo en confinamiento (sistema convencional). Este sistema se caracteriza por animales con alta eficiencia de producción, estabulados en alta densidad, alimentados sólo con concentrados, por tanto, podrían estar sometidos a mayor estrés (Edwards, 2005).

Una opción diferente al sistema convencional, es la producción en sistema extensivo o natural, también llamado "outdoor" o "free range", con acceso a pradera para pastoreo. Los animales son suplementados con concentrados para satisfacer sus necesidades nutricionales (Edwards, 2005). En el sistema convencional, de igual forma que en crianza natural, se entrega a los animales alimentos balanceados, que pueden incluir productos de origen vegetal, pero no presentan acceso a praderas (Rivero et al., 2013).

El sistema de crianza natural tiene como ventaja, en comparación al sistema convencional, un costo bajo en infraestructura, ya que los cerdos al estar en pradera solo requieren instalaciones para su resguardo en las noches, días de frío y lluvia, y una instalación especial para las pariciones. En cambio, en producción en confinamiento los animales deben permanecer en distintas instalaciones a lo largo de su periodo productivo (Edwards, 2005). El aporte nutricional entregado por la pradera puede ser de buen nivel para rumiantes, sin embargo, los cerdos al ser monogástricos, no son capaces de aprovechar la totalidad de los nutrientes que ésta aporta, por lo que se requiere suplementación (Agyekum and Nyachoti, 2017). Cabe destacar que en la alimentación de cerdos criados en sistema natural no se utilizan alimentos de origen animal, a diferencia de los cerdos en sistema intensivo convencional (SAG, 2010; Rivero et al., 2013).

Se ha encontrado que los rumiantes en pastoreo, presentan una mayor concentración de ácidos grasos insaturados en la leche y carne, que animales alimentados con ensilaje (Elgersma et al., 2004; Morales et al., 2012), debido al contenido de ácidos grasos insaturados que presentan los alimentos de origen vegetal (Mas et al., 2011; Alonso et al., 2012). Sin embargo, con respecto a cerdos de crianza natural, la información a nivel nacional es escasa, existiendo mayores antecedentes en otros países (Inserra et al., 2015; Álvarez-Rodríguez et al., 2016; Zhao et al., 2016).

En Chile, la producción de cerdos en sistema natural podría representar una opción más atractiva para el consumidor, con carne de mejor calidad nutricional, proveniente de animales criados bajo protocolos acordes con parámetros de bienestar animal. Por lo tanto, el objetivo de este estudio fue determinar la composición de la carne de cerdo criado en un sistema natural.

\section{MATERIALES Y MÉTODOS}

\section{Crianza de animales en sistema de natural}

Los cerdos del estudio eran mestizos de raza Landrace dominante, machos y hembras, los cuales tuvieron acceso a praderas a partir de los 15 días de vida. Además, se utilizó una alimentación de acuerdo con los requerimientos del animal desde su nacimiento hasta la faena (NRC, 1998).

En general, la dieta se basó en maíz chancado, afrecho de soya, harinilla de arroz, suplemento vitamínico, sal y calcio, en distintas proporciones, dependiendo de la etapa en la que se encontraba el animal. Las praderas estaban compuestas por gramíneas y leguminosas, predominando Trifolium repens, Lolium perenne, Lotus y Dactylis glomerata (Ruiz, 1996). Las praderas estuvieron a disposición de los animales durante todo el día, exceptuando días fríos de invierno y las noches, en los que fueron estabulados. Los lechones recién nacidos fueron vacunados con hierro (1 $\mathrm{mL}$ por animal), y la castración de machos se 
realizó previo a los 17 días. El destete se efectuó a los 40 días, y se realizó estabulación para la engorda final a base de concentrados durante los últimos 30 días.

\section{Muestras de carne}

Los animales se faenaron a los 6 a 7 meses de edad, con pesos cercanos a los $70-80 \mathrm{~kg}$, trasladándolos desde el predio de origen al módulo de acopio de faena móvil (TriVan, Seattle, USA) en Perquilauquén, $30 \mathrm{~km}$ al oeste de Parral (Resolución Sanitaria $\mathrm{N}^{\circ} 2696$ y $\mathrm{N}^{\circ}$ 557, Ministerio de Salud de la Región del Maule, Chile). El aturdimiento se realizó mediante insensibilización eléctrica de acuerdo al Código Sanitario para Animales Terrestres (OIE, 2016). Las canales de los cerdos faenados se almacenaron en una cámara de frío a $3 \pm 1^{\circ} \mathrm{C}$. El desposte se realizó $24 \mathrm{~h}$ posteriores a la faena, obteniéndose muestras de lomo a nivel de la décima costilla, y de pulpa de pierna. Para los análisis se utilizaron 54 lomos y 54 pulpas de cerdos provenientes de sistema natural.

\section{Determinación de $\mathrm{pH}$}

El pH de la carne se determinó en el lomo a nivel de la décima costilla, y en la pierna, inmediatamente después de la faena y a las 24 $\mathrm{h}$ después del sacrificio. Se utilizó un pH-metro portátil (Hanna Instruments ATC modelo HI 8424, Portugal) con electrodo de penetración, previamente calibrado con buffer $\mathrm{pH} 4,0$ y 7,0. Las mediciones se realizaron en triplicado.

\section{Análisis proximal}

La composición nutricional de las muestras de carne se determinó en el Laboratorio de Nutrición Animal de la Universidad de Concepción, Chillán, Chile.

Para la determinación de materia seca, las muestras de carne se sometieron a una molienda, seguido de congelación $\left(-18^{\circ} \mathrm{C}\right)$, y liofilización a $-65^{\circ} \mathrm{C}$ por $72 \mathrm{~h}$ (Christ Alpha 1-4 LD Plus, Osterode, Germany). Las muestras secas se sometieron a una molienda fina utilizando un homogenizador (Thomas Scientific, modelo 3383-L40, Swedesboro, New Jersey, USA) y se mantuvieron congeladas en bolsas plásticas herméticas para realizar los otros análisis.

Se determinó el contenido de proteína cruda (método 981.10), extracto etéreo (método 960.39), y cenizas totales (método 920.153) (AOAC, 2005). La determinación de sodio se realizó mediante lectura directa por espectrofotometría de emisión atómica, con espectrómetro (SOLAAR 969 AA MkII, Inglaterra), a una longitud de onda de 589 nm.

\section{Perfil de ácidos grasos}

El análisis del perfil de ácidos grasos de la carne y grasa de cobertura se realizó en el Laboratorio de Química de Alimentos de la Universidad del Bío-Bío (Chillán, Chile).

Las muestras liofilizadas se sometieron a una extracción de grasa utilizando el método Soxhlet (método 920.39) (AOAC, 2005). El residuo sólido se utilizó en la determinación de los ácidos grasos libres, después de la formación de metil ésteres de ácidos grasos. La solución estándar de ácidos grasos correspondió a una mezcla de ácidos grasos de $100 \mathrm{mg} \mathrm{mL}^{-1}$ (Sigma Chemical Co., St. Louis, Missouri, USA), la cual se diluyó con hexano a $10 \mathrm{mg} \mathrm{mL}^{-1}$. La determinación del perfil de ácidos grasos se realizó a través del método cromatográfico (método 996.06) (AOAC, 2005), utilizando un cromatógrafo de gases (Agilent Technologies, $6890 \mathrm{~N}$, USA) y una columna de 60 $\mathrm{m} \times 0,25 \mathrm{~mm}$ ID, 0,15 $\mu \mathrm{m}$ (Agilent Technologies J\&W DB-23, USA). Las condiciones de operación fueron las siguientes: temperatura del inyector y detector a $250^{\circ} \mathrm{C}$; temperatura a $120^{\circ} \mathrm{C}$ por $5 \mathrm{~min}$, con incremento hasta $240^{\circ} \mathrm{C}$ a una velocidad de $4^{\circ} \mathrm{C} \mathrm{min}^{-1}$ por $30 \mathrm{~min}$. La temperatura final se mantuvo por $25 \mathrm{~min}$. La velocidad de flujo del gas portador (nitrógeno) fue de $1 \mathrm{~mL} \mathrm{~min}{ }^{-1}$. La relación de división fue de 20:1 y la velocidad lineal del nitrógeno fue de $28,5 \mathrm{~cm} \mathrm{~s}^{-1}$ a $120^{\circ} \mathrm{C}$.

\section{Análisis estadístico}

Para la comparación de $\mathrm{pH}$ y composición de lomo y pulpa de pierna se utilizó la prueba t de Student con un nivel de significancia del 5\%. Para el contenido de ácidos grasos de lomo, pulpa de pierna y grasa de cobertura, se realizó un análisis de varianza con un nivel de significancia del $5 \%$, y para la comparación de medias se aplicó el test de Tukey $(\mathrm{P} \leq 0,05)$, utilizando el programa estadístico INFOSTAT ${ }^{\circledR}$.

\section{RESULTADOS Y DISCUSIÓN}

Los valores de $\mathrm{pH}$ inicial de la carne después de la faena y a las $24 \mathrm{~h}$ post mortem se presentan en la Tabla 1. El pH promedio de la carne de cerdos de sistema natural al momento de faena fue de 6,39 para lomo, y 6,37 para pulpa de pierna ( $\mathrm{P}>$ $0,05)$, disminuyendo a las 24 horas post mortem a valores de 5,81 y 5,70, respectivamente $(\mathrm{P} \leq 0,05)$, producto de la formación de ácido láctico en condiciones anaeróbicas post mortem (Scheffler et al., 2013). El pH obtenido para lomo y pierna se encuentran cercanos a resultados obtenidos por Lindahl et al. (2006) y Jama et al. (2016), quienes obtuvieron valores de $\mathrm{pH}$ cercanos a 5,5 en carne de cerdo a las $24 \mathrm{~h}$ después de la faena. El pH de la carne de cerdo generalmente fluctúa entre 5,3 a 
Tabla 1. pH inicial de carne de cerdo natural después de la faena y a 24 h post mortem.

Table 1. Initial $\mathrm{pH}$ of natural pork meat after slaughtering $\left(\mathrm{t}_{0}\right)$ and at $24 \mathrm{~h}\left(\mathrm{t}_{24}\right)$ post mortem.

\begin{tabular}{lcc}
\hline Corte de carne & $\mathbf{t}_{0}$ & $\mathbf{t}_{24}$ \\
\hline Lomo & $6,39 \pm 0,56$ aA & $5,81 \pm 0,26 \mathrm{aB}$ \\
Pulpa de pierna & $6,37 \pm 0,28$ aA & $5,70 \pm 0,15 \mathrm{bB}$ \\
\hline
\end{tabular}

Medias con letras minúsculas iguales no presentan diferencias significativas entre cortes de carne $(\mathrm{P}>0,05)$. Medias con letras mayúsculas iguales no presentan diferencias significativas entre tiempos $(\mathrm{P}>0,05)$.

6,0 a las $24 \mathrm{~h}$ post mortem (Scheffler et al., 2013), por lo que los valores encontrados reflejarían un nivel de estrés normal de los cerdos de este estudio. A las $24 \mathrm{~h}$ post mortem el $\mathrm{pH}$ de la pierna fue levemente inferior al $\mathrm{pH}$ del lomo $(\mathrm{P} \leq 0,05)$, lo que podría deberse a un contenido levemente superior de glucógeno o una transformación más rápida de glucógeno a ácido láctico en la pierna.

El contenido de materia seca de la carne fue cercano al 30\% (Tabla 2), siendo levemente mayor en pulpa de pierna $(\mathrm{P} \leq 0,05)$, al presentar este corte menor contenido de humedad. No se presentaron diferencias significativas en los otros componentes analizados en la carne de cerdo $(\mathrm{P}>0,05)$. En cuanto a los valores obtenidos de proteínas, éstos concuerdan con valores cercanos al 20\%, informados por Aaslyng et al. (2003), Tornberg et al. (2005), y Becker et al. (2016). Los valores de extracto etéreo obtenidos son superiores a los reportados por otros autores, los que coinciden en valores cercanos al $3 \%$ en cerdos de 73 a $79 \mathrm{~kg}$ de peso (Aaslyng et al., 2003; Tornberg et al., 2005; Becker et al., 2016).

El perfil de ácidos grasos de lomo, pulpa de pierna y grasa de cobertura de cerdos del sistema natural se presenta en la Tabla 3. En todos se presentó un mayor contenido de ácido oleico $(18: 1 \mathrm{n} 9 \mathrm{c})$, seguido de ácido palmítico $(16: 0)$ y esteárico $(18: 0)(P \leq 0,05)$. En lomo y pulpa de pierna el contenido de ácidos grasos monoinsaturados fue mayor que saturados y poliinsaturados $(\mathrm{P} \leq 0,05)$. Otros estudios también han encontrado mayores contenidos de ácido oleico (18:1n9c), ácido palmítico (16:0) y esteárico (18:0), y mayor contenido de ácidos grasos monoinsaturados en lomo de cerdos de sistema orgánico y convencional (ÁlvarezRodríguez et al., 2016; Janiszewski et al., 2016). Al comparar entre cortes, la carne presentó un mayor contenido de los ácidos grasos mirístico (14:0), palmitoleico (16:1), linoeládico (18:2n6t), y menor contenido de los ácidos esteárico (18:0) y araquidónico (20:0), que la grasa de cobertura $(\mathrm{P} \leq 0,05)$. En el lomo se encontró un contenido mayor de ácido palmítico (16:0), oleico (18:1n9c) y eicosenoico (20:1), y un contenido menor de ácido linoleico (18:2n6c) y $\alpha$-linolénico (18:3n3), comparado con pulpa de pierna $(\mathrm{P} \leq 0,05)$.

En la carne, el contenido de ácidos grasos saturados y monoinsaturados fue mayor, y el de poliinsaturados menor que en la grasa de cobertura $(P \leq 0,05)$. Esto se podría deber al consumo de forrajes verdes desde los inicios de su desarrollo, ya que la grasa subcutánea presenta una tasa de renovación más lenta que la grasa intramuscular (Jiang et al., 2018). En este estudio los animales estuvieron estabulados sin acceso a pradera el último mes, lo cual podría haber influido en la composición final de la grasa infiltrada en la carne. Por lo tanto, para aumentar los ácidos grasos monoinsaturados y poliinsaturados en la carne de cerdos de sistema natural, sería recomendable prolongar el pastoreo hasta el final del proceso productivo (Jiang et al., 2018). Álvarez-Rodríguez et al. (2016) determinaron un mayor contenido de ácidos grasos poliinsaturados en carne de cerdos de sistema orgánico, pero sin acceso a praderas, comparado con cerdos de sistema convencional, lo que se atribuye a ácidos grasos contenidos en la soya, como el $\alpha$-linolénico (18:3n3).

Con los resultados obtenidos en este estudio se recomienda evaluar la calidad de carne de animales criados en sistema natural que tengan una fase de término en pradera, y comparar con animales de iguales características de un sistema convencional sin acceso a pradera.

\section{CONCLUSIONES}

De acuerdo a los resultados obtenidos en este estudio, se puede concluir que la carne de cerdo producido en un sistema natural se caracteriza por presentar un $\mathrm{pH}$ a las $24 \mathrm{~h}$ post mortem en pulpa de pierna levemente inferior al $\mathrm{pH}$ del lomo, un mayor contenido de humedad en el lomo que en la pulpa de pierna, un perfil de ácidos grasos compuesto principalmente por ácido oleico (18:1n9c), palmítico (16:0) y esteárico (18:0), y un menor contenido de ácidos grasos poliinsaturados que la grasa subcutánea. 
Tabla 2. Composición nutricional de carne de cerdos criados en sistema natural.

Table 2. Nutritional composition of pork meat from pigs raised in a natural production system.

\begin{tabular}{lrr}
\hline Composición & \multicolumn{2}{c}{ Pulpa de pierna } \\
\hline Materia seca, \% & $28,88 \pm 2,10 \mathrm{a}$ & $29,58 \pm 2,18 \mathrm{~b}$ \\
Proteínas, \%* & $74,36 \pm 3,85 \mathrm{a}$ & $72,84 \pm 6,72 \mathrm{a}$ \\
Grasa, \%* & $15,67 \pm 3,33 \mathrm{a}$ & $16,61 \pm 6,67 \mathrm{a}$ \\
Minerales, \%* & $4,17 \pm 0,29 \mathrm{a}$ & $4,27 \pm 0,43 \mathrm{a}$ \\
Sodio, mg 100 g ${ }^{-1 *}$ & $207,97 \pm 34,2 \mathrm{a}$ & $217,30 \pm 22,7 \mathrm{a}$ \\
\hline
\end{tabular}

${ }^{*}$ En base seca. Medias con letras iguales no presentan diferencias significativas $(\mathrm{P}>0,05)$.

Tabla 3. Perfil de ácidos grasos en carne y grasa de cobertura de cerdos criados en sistema natural. Table 3. Fatty acids profile in pork meat and cover fat from pigs raised in a natural production system.

\begin{tabular}{lrrr}
\hline \multicolumn{1}{c}{ Ácidos grasos } & \multicolumn{1}{c}{ Lomo } & \multicolumn{1}{c}{ Pulpa de pierna } & \multicolumn{1}{c}{ Grasa } \\
\hline Mirístico (14:0) & $1,39 \pm 0,40 \mathrm{cB}$ & $1,26 \pm 0,31 \mathrm{~dB}$ & $0,92 \pm 0,16 \mathrm{fA}$ \\
Palmítico (16:0) & $28,65 \pm 1,61 \mathrm{gB}$ & $25,93 \pm 1,56 \mathrm{gA}$ & $26,47 \pm 5,21 \mathrm{iA}$ \\
Palmitoleico (16:1) & $3,71 \pm 0,84 \mathrm{eB}$ & $3,69 \pm 1,47 \mathrm{eB}$ & $1,19 \pm 0,16 \mathrm{gA}$ \\
Margárico (17:0) & $0,33 \pm 0,13 \mathrm{aA}$ & $0,42 \pm 0,17 \mathrm{bA}$ & $0,37 \pm 0,07 \mathrm{dA}$ \\
Margaroleico (17:1) & $0,20 \pm 0,05 \mathrm{aA}$ & $0,20 \pm 0,05 \mathrm{aA}$ & $0,20 \pm 0,04 \mathrm{cA}$ \\
Esteárico (18:0) & $14,28 \pm 2,19 \mathrm{fA}$ & $14,85 \pm 1,19 \mathrm{fA}$ & $18,84 \pm 4,19 \mathrm{jB}$ \\
Oleico (18:1n9c) & $48,56 \pm 2,29 \mathrm{hB}$ & $43,31 \pm 5,10 \mathrm{hA}$ & $40,78 \pm 4,26 \mathrm{kA}$ \\
Linoeládico (18:2n6t) & $0,23 \pm 0,22 \mathrm{aB}$ & $0,25 \pm 0,18 \mathrm{aB}$ & $0,04 \pm 0,02 \mathrm{aA}$ \\
Linoleico (18:2n6c) & $1,77 \pm 0,78 \mathrm{dA}$ & $3,79 \pm 1,08 \mathrm{eB}$ & $15,82 \pm 2,54 \mathrm{jC}$ \\
$\alpha$-Linolénico (18:3n3) & $0,12 \pm 0,06 \mathrm{aA}$ & $0,17 \pm 0,06 \mathrm{aB}$ & $0,10 \pm 0,02 \mathrm{bA}$ \\
Araquidónico (20:0) & $0,20 \pm 0,05 \mathrm{aA}$ & $0,21 \pm 0,04 \mathrm{aA}$ & $0,57 \pm 0,21 \mathrm{eB}$ \\
Eicosenoico (20:1) & $1,00 \pm 0,21 \mathrm{bB}$ & $0,76 \pm 0,40 \mathrm{cA}$ & $1,51 \pm 0,38 \mathrm{hC}$ \\
\hline Saturados & $44,84 \pm 2,59 \mathrm{bB}$ & $42,60 \pm 1,72 \mathrm{bAB}$ & $40,89 \pm 9,80 \mathrm{bA}$ \\
Monoinsaturados & $53,24 \pm 2,19 \mathrm{cC}$ & $47,96 \pm 4,28 \mathrm{cB}$ & $43,72 \pm 4,43 \mathrm{bA}$ \\
Poliinsaturados & $2,12 \pm 0,87 \mathrm{aA}$ & $4,21 \pm 1,18 \mathrm{aB}$ & $15,96 \pm 2,55 \mathrm{aC}$ \\
\hline
\end{tabular}

Medias con letras minúsculas iguales no presentan diferencias significativas entre ácidos grasos $(\mathrm{P}>0,05)$. Medias con letras mayúsculas iguales no presentan diferencias significativas entre cortes de carne $(P>0,05)$.

\section{RECONOCIMIENTOS}

Financiamiento otorgado por el Fondo de Innovación para la Competitividad (FIC) Región del Maule (30.136.467-0) y la empresa Carnes Andes Sur S.A. (Parral, Chile).

\section{LITERATURA CITADA}

Aaslyng, M.D., C. Bejerholm, P. Ertbjerg, H.C. Bertram, and H.J. Andersen. 2003. Cooking loss and juiciness of pork in relation to raw meat quality and cooking procedure. Food Qual. Prefer. 14(4):277-288.

Agyekum, A.K., and C.M. Nyachoti. 2017. Nutritional and metabolic consequences of feeding high-fiber diets to swine: a review. Engineering 3:716-725.
Alonso, V., L.M. Najes, L. Provincial, E. Guillén, M. Gil, P. Roncalés, et al. 2012. Influence of dietary fat on pork eating quality. Meat Sci. 92(4):366-373.

Álvarez-Rodríguez, J., D. Villalba, D. Cubiló, D. Babot, and M. Tor. 2016. Organic practices and gender are effective strategies to provide healthy pork loin. J. Integr. Agric. 15(3):608617.

AOAC. 2005. Official methods of analysis. 18th. ed. Association of Official Analytical Chemists (AOAC) International, Gaithersburg, USA.

ASPROCER. 2019. Análisis sectorial. Asociación Gremial de Productores de Cerdos de Chile [en línea]. <https://www.asprocer.cl/ industria/analisis-sectorial/> [Consulta: 03 junio 2019]. 
Becker, A., A. Boulaaba, S. Pingen, C. Krischek, and G. Klein. 2016. Low temperature cooking of pork meat - Physicochemical and sensory aspects. Meat Sci. 118:82-88.

Edwards, S.A. 2005. Product quality attributes associated with outdoor pig production. Livest. Prod. Sci. 94(1-2):5-14.

Elgersma, A., G. Ellen, H. van der Horst, H. Boer, P.R. Dekker, and S. Tamminga. 2004. Quick changes in milk fat composition from cows after transition from fresh grass to a silage diet. Anim. Feed. Sci. Technol. 117(1-2):13-27.

Inserra, L., G. Luciano, M. Bella, M. Scerra, C. Cilione, P. Basile, et al. 2015. Effect of including carob pulp in the diet of fattening pigs on the fatty acid composition and oxidative stability of pork. Meat Sci. 100:256261.

Jama, N., V. Maphosa, L.C. Hoffman, and V. Muchenje. 2016. Effect of sex and time to slaughter (transportation and lairage duration) on the levels of cortisol, creatine kinase and subsequent relationship with pork quality. Meat Sci. 116:43-49.

Janiszewski, P., E. Grześkowiak, D. Lisiak, B. Broys, K. Borzuta, E. Pospiech, et al. 2016. The influence of thermal processing on the fatty acid profile of pork and lamb meat fed diet with increased levels of unsaturated fatty acids. Meat Sci. 111:161-167.

Jiang, Q., C. Li, Y. Yu, Y. Xing, D. Xiao, and B. Zhang. 2018. Comparison of fatty acid profile of three adipose tissues in Ningxiang pigs. Anim. Nutr. 4:256-259.

Lindahl, G., P. Henckel, A.H. Karlsson, and H.J. Andersen. 2006. Significance of early postmortem temperature and $\mathrm{pH}$ decline on colour characteristics of pork loin from different crossbreeds. Meat Sci. 72(4):613623.

Mas, G., M. Llavall, D. Coll, R. Roca, I. Díaz, M.A. Oliver, et al. 2011. Effect of an elevated monounsaturated fat diet on pork carcass and meat quality traits and tissue acid composition from York-crossed barrows and gilts. Meat Sci. 89(4):419-425.
Morales, R., C. Folch, S. Iraira, N. Teuber, and C.E. Realini. 2012. Nutritional quality of beef produced in Chile from different production systems. Chilean J. Agric. Res. 72(1):80-86.

NRC. 1998. Nutrient Requirements of Swine. 10th Revised Ed.. National Research Council (NRC), The National Academy Press. Washington, DC, USA.

ODEPA. 2019. Boletín de carne bovina. Oficina de Estudios y Políticas Agrarias, Gobierno de Chile [en línea]. <https://www.odepa.gob.cl/ wp-content/uploads/2019/02/Bcarne022019. pdf $>$. [Consulta: 03 junio 2019].

OIE. 2016. Código Sanitario para los Animales Terrestres. Organización Mundial de Sanidad Animal, Francia [en línea]. <http:// www.oie.int/es/normas-internacionales/ codigo-terrestre/acceso-en-linea/>. [Consulta 16 noviembre 2016].

Rivero, J., I. López, and S. Hodgkinson. 2013. Pasture consumption and grazing behavior of European wild boar (Sus scrofa L.) under continuous and rotational grazing systems. Livest. Sci. 154(1-3):175-183.

Ruiz, I. 1996. Praderas para Chile. 2a. ed. INIA, Santiago, Chile.

SAG. 2010. Instructivo: Planteles de animales porcinos bajo certificación oficial I-PP-IT-019. Servicio Agrícola y Ganadero, Gobierno de Chile [en línea]. <http://www.sag.gob.cl/ sites/default/files/I-PP-IT-019_porcinos.pdf >. [Consulta: 03 junio 2019].

Scheffler, T.L., J.M. Scheffler, S.C. Kasten, A.A. Sosnicki, and D.E. Gerrard. 2013. High glycolytic potential does not predict low ultimate $\mathrm{pH}$ in pork. Meat Sci. 95(1):85-91.

Tornberg, E. 2005. Effects of heat on meat proteins - Implications on structure and quality of meat products. Meat Sci. 70(3):493-508.

Zhao, Y., D. Wang, and S. Yang. 2016. Effect of organic and conventional rearing system on the mineral content of pork. Meat Sci. 118:103-107. 